OPEN ACCESS

Edited by:

Sergio Machado,

Salgado de Oliveira University, Brazil

Reviewed by:

Robinson Ramírez-Vélez, Universidad Pública de Navarra,

Spain

Liye Zou,

Shenzhen University, China

*Correspondence:

Haney Aguirre-Loaiza

haney.aguirre@ucp.edu.co

Specialty section:

This article was submitted to

Movement Science and Sport

Psychology,

a section of the journal

Frontiers in Psychology

Received: 01 June 2019 Accepted: 25 November 2019

Published: 20 December 2019

Citation:

Aguirre-Loaiza H, Arenas J,

Arias I, Franco-Jímenez A,

Barbosa-Granados $S$,

Ramos-Bermúdez S, Ayala-Zuluaga $F$ Núñez C and García-Mas A (2019)

Effect of Acute Physical Exercise on Executive Functions and Emotional Recognition: Analysis of Moderate to High Intensity in Young Adults.

Front. Psychol. 10:2774 doi: 10.3389/fpsyg.2019.02774

\section{Effect of Acute Physical Exercise on Executive Functions and Emotional Recognition: Analysis of Moderate to High Intensity in Young Adults}

\author{
Haney Aguirre-Loaiza ${ }^{*}$, Jaime Arenas², lanelleen Arias², Alejandra Franco-Jímenez ${ }^{3}$, \\ Sergio Barbosa-Granados ${ }^{4}$, Santiago Ramos-Bermúdez ${ }^{3}$, Federico Ayala-Zuluaga ${ }^{3,5}$, \\ César Núñez ${ }^{6}$ and Alexandre García-Mas ${ }^{7}$ \\ ${ }^{1}$ Department of Psychology, Catholic University of Pereira, Pereira, Colombia, ${ }^{2}$ Physical Education, University of Quindío, \\ Armenia, Colombia, ${ }^{3}$ Research Group Physical Activity, Cumanday, Manizales, Colombia, ${ }^{4}$ Psychology, Cooperative \\ University of Colombia, Pereira, Colombia, ${ }^{5}$ Department of Physical Action, Caldas University, Manizales, Colombia, \\ ${ }^{6}$ Psychology Program, Universidad de Medellin, Medellin, Colombia, ${ }^{7}$ Department of Basic Psychology, University of the \\ Balearic Islands, Palma, Spain
}

Physical exercise (PE) is associated with cognitive changes and brain function. However, it is required to clarify the effect of $\mathrm{PE}$ in different intensities, population groups conditions and the EF duration over different cognitive domains. Besides, no studies are known to have evaluated the contextual emotional recognition. Therefore, we studied the effect of acute PE of moderate intensities up to higher ones to the executive functions and the contextual emotional recognition. The participants were evaluated and classified in two experiments according to the IPAQ short form self-report and control measures. In both experiments, the groups were randomized, controlled, and exposed to one session of indoor cycling through intervals of high measure intensity (75-85\% HRmax). Experiment 1 comprised young adults who were physically active (PA) and healthy, apparently $\left(n=54, M_{\text {age }}=20.7, S D=2.5\right)$. Experiment 2 involved young adults who were physically inactive (IP) and healthy, apparently $\left(n=36, M_{\text {age }}=21.6, S D=1.8\right)$. The duration was the only factor that varied: $45 \mathrm{~min}$ for $\mathrm{PA}$ and $30 \mathrm{~min}$ for $\mathrm{PI}$. The executive functions were evaluated by the Stroop, TMT A/B, and verbal fluency, and the emotional recognition through a task that includes body and facial emotions in context, simultaneously. The analysis of factorial mixed ANOVA showed effects on the right choices of the indoor cycling groups in the PA, and the time response in PI. Also, other effects were observed in the controlled groups. TMT-A/B measures showed changes in the pre-test-post-test measures for both experiments. Verbal fluency performance favored the control group in both experiments. Meanwhile, the emotional recognition showed an effect of the PE in error-reduction and enhanced the scores in the right choices of body emotions. These results suggest that the EF with intensities favored cognitive processes such as inhibitory control and emotional recognition in context. We took into account the importance of 
high-complexity tasks design that avoid a ceiling effect. This study is the first on reporting a positive effect of PE over the emotional contextual recognition. Important clinical and educational implications are presented implications which highlight the modulatory role of EF with moderate to high intensities.

Keywords: physical exercise, cognitive neuroscience, cognitive performance, executive functions, emotional recognition, exercise psychology

\section{INTRODUCTION}

Physical exercise (PE) is an important environmental factor with positive effects on the brain and healthy behavior along different life stages (Herting et al., 2014; Hillman et al., 2014; Etnier et al., 2019), mainly when considering that less than $60 \%$ of world population does not do required PE (World Health Organization [WHO], 2018, World Health Organization [WHO], 2007). Such data, nowadays, are increasing and associated with sedentary lifestyles and low PE involvement (Guthold et al., 2018; Päivärinne et al., 2018). Thus, PE is considered a nonpharmacological strategy that has direct effects in functional and cognitive brain structures (Herting et al., 2014; Erickson et al., 2015; Prakash et al., 2015; Voss et al., 2015; Hagger, 2019). Researchers have addressed the questionings of the effect of EF on cognition from two perspectives. On the one hand, chronic effects in PE (e.g., weeks, months, and/or years). On the other hand, the immediate effect of the acute PE (e.g., one session). This study focuses on the second perspective, treating the effect of acute PE on cognitive processes and emotional recognition.

Most of the reviewed literature matches on observing positive changes of cognition after PE (Reed and Ones, 2006; Lambourne and Tomporowski, 2010; Chang et al., 2012; Donnelly et al., 2016; Ludyga et al., 2016; Sáez de Asteasu et al., 2017; Esteban-Cornejo et al., 2019; Etnier et al., 2019; Hillman et al., 2019; McSween et al., 2019). Likewise, it has been demonstrated that an acute PE brings benefits for some ways of performance on cognitive tasks after exercise. By the same token, executive functions improve after PE (Wu et al., 2019). Recent studies, however, affirm that there is not enough evidence that $\mathrm{PE}$ is positive for cognition or brain function and structure in child and young samples (Gunnell et al., 2018). What explains these possible contradictions is the huge methodological variety, and therefore, new investigative contributions might clarify and provide evidence of the relation of PE on cognition (Pontifex et al., 2019).

The relation between $\mathrm{PE}$, cognition, brain functions, and structures seem to rest upon several factors (Chang et al., 2012, 2015; Erickson et al., 2015; Gunnell et al., 2018). For instance, duration, intensity, and PE modality, as well as the vital cycle, the type of cognitive performance and physical condition of the participant. Even the effect of PE can vary depending on the cognitive domain (Pontifex et al., 2009, 2019). These factors are relevant in questioning the relation between $\mathrm{PE}$, brain and cognition. Hence, further research is required that identifies its modulating role (Labelle et al., 2013; Ludyga et al., 2016).

Addressing the related factors of PE (e.g., type, intensity, and duration), some contributions have pointed out that aerobic $\mathrm{PE}$ can improve cognitive performance in both young and old adults (Chang et al., 2012; Joyce et al., 2014; Tsukamoto et al., 2016). Specifically, Physiological changes have been observed by activating circuits of the prefrontal and occipital cortex in tasks that include a great cognitive effort (e.g., attention and executive control) (Chen and Chang, 2012; Basso et al., 2015). Other studies have reported that aerobic PE can enhance cognitive and neurological function (Armstrong and Welsman, 2007; Herting et al., 2014; Li et al., 2014; Reiter et al., 2015). Likewise, aerobic $\mathrm{PE}$ is associated with improved memory efficiency (Herting and Nagel, 2013; Voss et al., 2019) and inhibitory control performance (Pontifex et al., 2019). Even participants who were exposed to the multimodal combination (training of aerobic PE followed by cognitive training) showed improvement in cognitive performance as compared to those who only had a cognitive training (Wang et al., 2019a).

The immediate effects of $\mathrm{PE}$ on cognitive performance seem to be conditional on intensity of PE. Inverted $U$ hypothesis (Gutin, 1973) supports that cognitive performance tends to have more benefits after a moderate intensity of aerobic PE $(>64 \%$ Maximum heart rate - HRmax) in contrast to low ones $(<50 \%$ HRmax) or high intensities ( $>80 \%$ HRmax). In this regard, the evidence which supports the inverted $U$ hypothesis is wide (Pontifex et al., 2009; Hillman et al., 2014; Li et al., 2014; De Souto Barreto et al., 2016; Ludyga et al., 2016). Nonetheless, current studies inform that high intensities of PE allow to have benefits in cognitive domains as inhibitory control (Quintero et al., 2018), memory and metacognition (Zuniga et al., 2019). Further, it is important to underscore that the number of studies of $\mathrm{PE}$ interventions of moderate intensity is actually small compared to interventions of moderate or low intensities (Browne et al., 2017). There are even more limited studies that address the transition of moderate and high intensities.

As a matter of fact, there is no agreement on the effect of duration, type or intensity of PE on cognition and the different types of cognitive domains (Vazou et al., 2019). A line of research suggested by other authors consists of clarifying the effects of PE in people who are physically active (PA), inactive and/or sedentary. Moreover, it associates more cognitive domains (Sink et al., 2015; Tsukamoto et al., 2016; Vieira et al., 2016; Browne et al., 2017; Peruyero et al., 2017; Silva et al., 2019). Based on the above-mentioned, narrative, systematic, and metaanalytic revisions have suggested an improvement of cognitive performance after an acute session of PE (Lambourne and Tomporowski, 2010; Chang et al., 2012; Ludyga et al., 2016; Basso and Suzuki, 2017; McSween et al., 2019); yet, constructs such as emotional recognition must be studied in relation to acute PE.

In that way, understanding how environmental factors, such as $\mathrm{PE}$, contribute to the performance of emotional recognition in 
context, and in ecological situations, is a novel area that answers to current questionings of social and cognitive neuroscience (Aviezer et al., 2008; Kumfor et al., 2018). We assumed that acute $\mathrm{PE}$ has positive effects on emotional recognition. Especially, whenever the aerobic exercise is combined with the demands of coordination seems that it gets more benefits on facial emotion recognition (Brand et al., 2019). In fact, the capacity of information processing, which links body movements with body-face recognition (Kumfor et al., 2018), is associated with brain regions such as motor areas, cerebellum, and fusiform gyrus (Committeri et al., 2007; Yogev-Seligmann et al., 2008), that are connected with the acute PE (Li et al., 2014; Esteban-Cornejo et al., 2019; Won et al., 2019). Recent paradigms on emotional recognition have located body information as contextual keys, taking away attention from facial recognition processing (Aviezer et al., 2008, 2012).

Based on the described evidence, that the dose-response relation in terms of intensity, duration and the type of acute PE over cognitive performance, specifically with executive functional tasks and emotional recognition, is an issue that needs to be studied. By solving the previous matter, we would contribute with knowledge about the strategy of PE, which can be a potent and promising environmental source that allows to adjust cognitive processes (executive performance to be precise) and emotional recognition. Among other scopes, it would facilitate the capacity of social human interactions in clinical and academic activities that improve the mental health of people. Thus, the main objective is to study the effect of acute $\mathrm{PE}$ of indoor cycling with a range of moderate and high intensity (75-85\% HRmax) in regard to executive functions and emotional recognition in PA and inactive people and high apparently.

\section{MATERIALS AND METHODS}

\section{Design and Participants}

Two experiments were designed, controlled and randomized with pre-test-post-test measures, for an indoor cycling session. The process of choosing, randomizing, and selecting is shown in Figure 1. Initially, $(n=140)$ were recruited, and 90 university students were analyzed $\left(M_{\text {age }}=21.0, D E=2.3\right)$. In both experiments, people were randomized by a computer-generated list through Excel (function RANDBETWEEN) from 1 to 60 in PA participants, and between 1 and 53 in physically inactive. The first experiment was carried out in young adults who were PA, and apparently healthy $(n=54)$. La They were randomly assigned to the experimental group and were exposed to a $45 \mathrm{~min}$ session of indoor cycling, whereas the control group had no PE. The second experiment involved physically inactive young adults $(n=36)$ who were randomly selected for the control and experimental groups. The indoor cycling session in the second experiment lasted $30 \mathrm{~min}$. The intensity of a session of $\mathrm{PE}$ was moderate-high ranging between 75 and 85\% HRmax. The intensity ranges are: moderate between 64 and $76 \%$ HRmax, and High 76-96\% HRmax (ACSM, 2018) The HRmax was calculated by Karvonen et al. (1957) 220-age (see section "Procedure").

\section{Measure}

\section{General Characteristics}

The participants answered a battery of instruments according to pre-experimental protocol (see section "Procedure"). Table 1 provides data such as age, body mass, resting heart rate (supine position for $10 \mathrm{~min}$ ), cognitive screening (MoCA), selfreport of physical activity (IAPQ), and Depression (BID). As expected, significative differences in the IPAQ self-report were observed $(p<0.000)$.

\section{Measurement Screening Cognitive screening}

The MoCA: Montreal Cognitive Assessment measures 10 cognitive domains with satisfactory results of sensitivity and specificity, which fluctuate between $82-90 \%$ and $75-87 \%$, respectively (Nasreddine et al., 2005). The MoCA is one of the most used instruments in clinics and research that identifies early cognitive changes. Cut-off points vary depending on educational and cultural level (O'Driscoll and Shaikh, 2017). Hence and having in mind Colombian population, one cut-off and exclusion point was assumed $<24$ (Pedraza et al., 2014, 2016; Gil et al., 2015).

The BDI - II: Beck Depression Inventory tests the existence or severity of depressive symptoms with 21 items. Spanish version was used (Sanz and García-Vera, 2013). The BID-II supports satisfactory psychometric evidence (Wang and Gorenstein, 2013). It was excluded such individuals whose score was $>30$ were excluded. The observation of depressive symptomatology can constitute a confusing factor, and thereupon, it was relevant to consider this control measure.

\section{Physical exercise measure}

The IPAQ-SF: The International Physical Activity Questionnaire - Short Form is used to facilitate a measurement and monitoring of physical activity (Craig et al., 2003), and seven questions about the last 7 days in relation to duration, minutes, hours, and days. A total measured score in METs (metabolic equivalent of task minutes per week) is recorded. The three classification levels are the following; low (e.g., walking), moderate (e.g., recreational cycling), and strong (e.g., intense aerobic activity). It is recognized as one of the most used questionnaires to determine AF level (Forsén et al., 2010; Van Poppel et al., 2010). The evidence of consistency and validity of the scores has been reported in several psychometric revisions (Sanda et al., 2017). The prior measurements are in line with the World Health Organization's global recommendations for physical activity (World Health Organization [WHO], 2007, 2010). IPAQ-SF was used with the aim of classifying active and inactive physically groups.

PARQ \& You: The Physical Activity Readiness Questionnaire has been an easy-to-use tool for people between 15 and 69 years of age. The PARQ \& You identifies health and cardiovascular problems from seven questions. It is usually applied to apparently healthy individuals, who have the willingness to take part in PE programs (Warburton et al., 2011). A participant's selfreport which was associated with a cardiovascular problem was excluded from the study. 


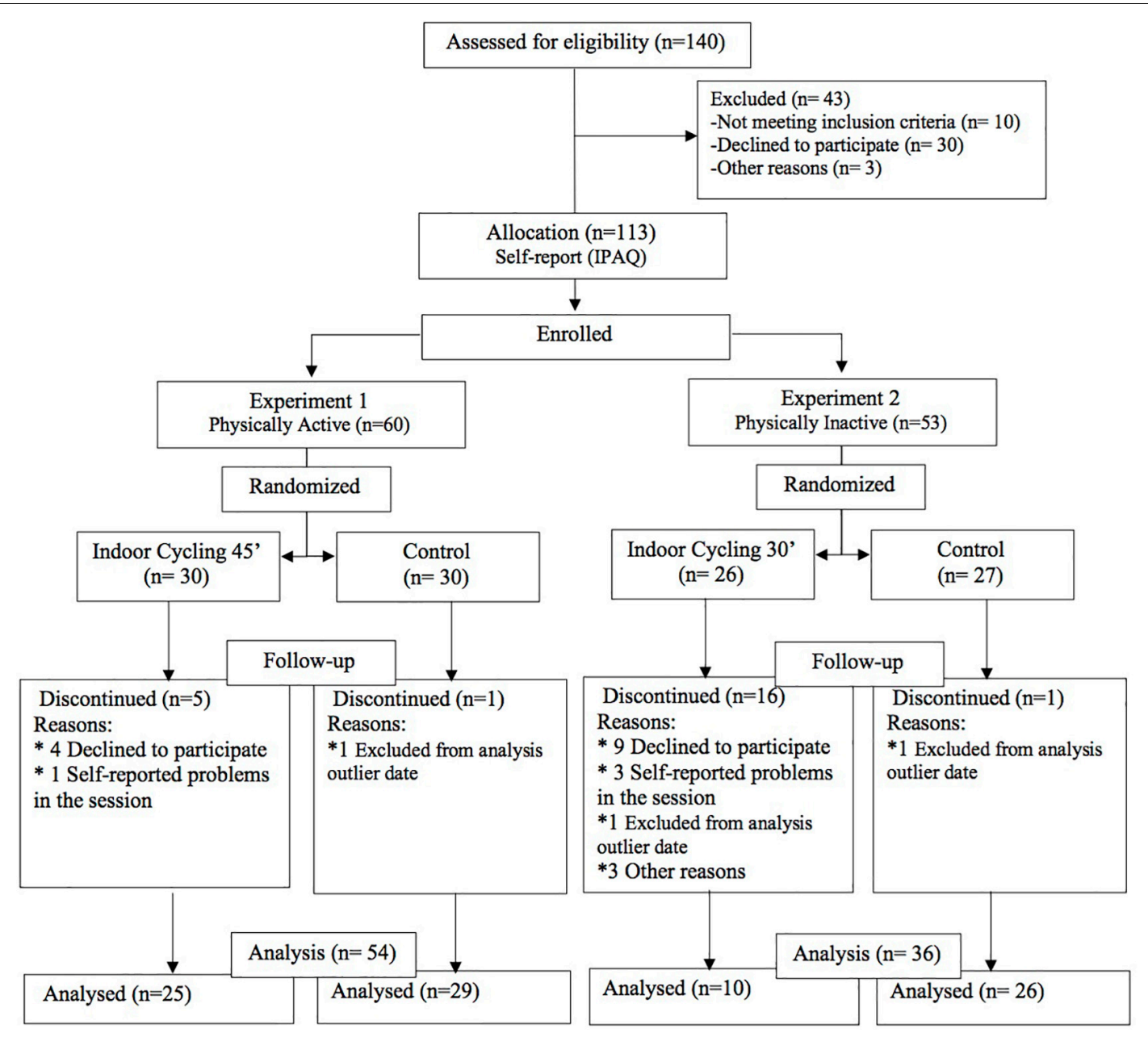

FIGURE 1 | Flow of participants throughout the study.

\section{Task of Executive Functions}

An executive functioning battery was registered, which is composed of three tasks; Stroop, TMT, and Verbal Fluency. These tasks are widely used in executive function measurement (Strauss et al., 2007). Moreover, other revisions have dealt with the PE effect on cognitive processes (Chang et al., 2012; Ludyga et al., 2016; McSween et al., 2019; Pontifex et al., 2019).

(a) Stroop: it evaluates the inhibitory control as executive process and it is associated with function in the anterior cingular cortex. An adapted version of the Stroop test version was performed in pencil and paper format by manipulating two conditions (Florez et al., 2012). In Stroop-A, the person reads written words, and as soon as it is highlighted, he/she must mention the color and avoid saying the word. In the Stroop-B, the evaluator points to the columns of words that are in color, and the individual reads what is written, but when the evaluator says the word "color," the person should mention the ink's color without naming the word. In both conditions, 84 stimuli were found. The response and the percentage of the right choices as well as answer time of the execution in both conditions was measured.

(b) The Trail Making Test (TMT) evaluates attention processes, visual scanning, mental processing, and flexibility (Reitan, 1958). It consists of two assessed parts in paper - pencil format. TMT-A deals with the sequence of ascending numbers from 1 to 25 . The individuals must match the locked-numbers. The TMT-B is used for the estimation of executive functions (e.g., attention, planning, cognitive flexibility, and response inhibition). The individual has to match the aligned circles randomly by alternating between upward numbers (1-3) and the letters (A-L) (1-A-2-B, etc.). Normally, the TMT-A and the TMT-B are valid and reliable measures (Rabin et al., 2005).

(c) Verbal Fluency (Phonemic and Semantic): It evaluates the capacity to access lexicon and recover semantic information under limited conditions, and its psychometric properties are satisfactory (Borkowski et al., 1967; Strauss et al., 2007). The individual must mention as many words as he/she can, that start with the letter "S" (phonological fluency). Regarding phonological fluency, he must mention as many 
TABLE 1 | Characteristics of participants.

\begin{tabular}{|c|c|c|c|c|c|c|}
\hline \multirow[t]{3}{*}{ Characteristics } & \multirow{2}{*}{\multicolumn{2}{|c|}{$\begin{array}{c}\text { Physically active }(n=54) \\
\text { Experiment } 1\end{array}$}} & \multirow{2}{*}{\multicolumn{2}{|c|}{$\frac{\text { Physically inactive }(n=36)}{\text { Experiment } 2}$}} & \multirow[t]{3}{*}{$t$} & \multirow[t]{3}{*}{$p$} \\
\hline & & & & & & \\
\hline & $M$ & $S D$ & $M$ & $S D$ & & \\
\hline Age (years) & 20.7 & 2.5 & 21.6 & 1.8 & -0.029 & 0.977 \\
\hline Body mass (kg) & 65.5 & 13.8 & 64.3 & 10.6 & 0.325 & 0.746 \\
\hline Resting heart rate (bpm) & 63.9 & 11.0 & 70.8 & 8.3 & -1.83 & 0.73 \\
\hline Physical activity - IPAQ (METs) & 6190.8 & 5087.1 & 176.8 & 195.6 & $7.07^{* *}$ & 0.000 \\
\hline Depression - BDI & 7.3 & 5.9 & 9.94 & 6.7 & -1.89 & 0.062 \\
\hline Cognitive screening - MoCA & 26.57 & 1.2 & 26.06 & 2.2 & 1.36 & 0.176 \\
\hline
\end{tabular}

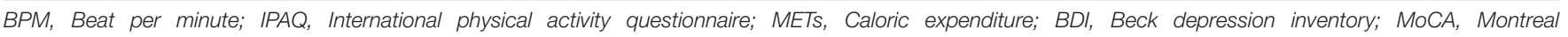
cognitive assessment. ${ }^{* *} p<0.01$.

words as possible in relation to a category or a common interest. We tested the "animals" category. In both tasks, $60 \mathrm{~s}$ were timed. Words from the same morphology or proper names were excluded. The total score is the sum of all the right words that are produced in each category.

\section{Task Emotional Recognition}

The experimental task of recognition of body-face emotions was used in context (see Figure 2). This paradigm was originally developed by Aviezer et al. (2008), and used in subsequent studies (Aviezer et al., 2011, 2012; SantamaríaGarcía et al., 2019). This task consists of the representation of four basic emotions (anger, sadness, fear, and displeasure), involving body context. Each emotion is represented by the facial expression along with the body context. In the same way, the facial and corporal emotions can be either congruent or incongruent (e.g., consistent, sadness-face, and sadness-body; inconsistent, sadness-face, and anger-body). We submitted a total of 80 stimuli (Height $11.4 \mathrm{~cm} \times$ Width $15.2 \mathrm{~cm}$ ) which were assigned randomly in a 14 " computer screen Microsoft Power Point. The task's command is "You have to select the emotional option that best describes the facial expression." Each participant had to choose the right answer from six options of basic emotions (sadness, anger, fear, disgust, happiness, and surprise). There is no time limit on task execution. The precision of correct response of the facial emotional expression was scored. The assigned scoring for the correct match of facial emotion $=2$ points. If the contextual influence matches the body emotion $=1$ point. When the person neither got the facial emotion, nor the body context right ( 0 points). The total score turned into a response rate (Response Accuracy $=$ Sum of score $/ 84 \times 100)$.

\section{Procedure}

The volunteers participated in three stages (pre-experimental session, experimental session, and post-experimental session). To maximize transparency and replicability, the indoor Cycling program described in this manuscript follows the Consensus on Exercise Reporting Template (CERT) (Slade et al., 2016) (see Supplementary Table S1).

\section{Pre-experimental Session}

In the pre-experimental session, the objectives and the scope were presented. In addition to the signature of the informed consent, information about the age, weight, HR at rest, MoCA, BDI-II, IAPQ-SF, and PARQ (see Table 1) was collected. All of these measures were obtained in the days before the indoor cycling PE session. According to the IAPQ-SF results, the participants were classified into one of the two experiments: Experiment 1 PA volunteers whose physical activity report was moderate and intense. Experiment 2 - physically inactive volunteers whose physical activity was in low category. The participants were instructed regarding clothing, hydration, and feeding before the PE session. The protocol of cognitive measures and emotional recognition from the pretest was executed from 45 up to $60 \mathrm{~min}$ before the experimental session in the fitness center. After the evaluation protocol, the instructor informed about the basic concepts, techniques and commands executed in the indoor cycling session. The instructor has a degree in Physical Education with international certification in Indoor Cycle and 10 years of experience in sports training.

\section{Experimental Manipulation}

Each experiment controlled the HR through Polar-M200, whose $\mathrm{HR}$ register is obtained by sensors from the wrist. The intensities of PE were moderates (64-76\% HRmax), and high (76-96\% HRmax) (ACSM, 2018). Data were gathered through easy connectivity between the device and the computer. The HRmax was calculated by the 220-Age formula. During the experimental session, a research assistant monitored HR behavior and the RPMs that corresponds to the training zone of each participant. The bicycles were standard and relevant to the indoor cycling aims.

Experiment 1 - Physically active group: The participants were randomly assigned to the experimental and control group (see Figure 1). The experimental group performed an indoor cycling session with intensities between 75 and 85\% HRmax for $45 \mathrm{~min}$ (see Supplementary Figure S1). The PE protocol had three phases. Phase 1, warming up and muscle activation (10 min) 60-65\% HRmax between 60 and 90 RPM (revolutions per minute). Phase 2, main work (35 min) which had six sections of 4 min with six peaks of $85 \%$ HRmax between 90 
A

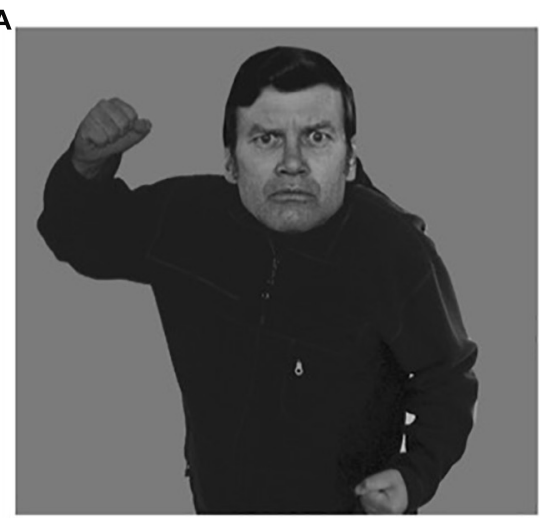

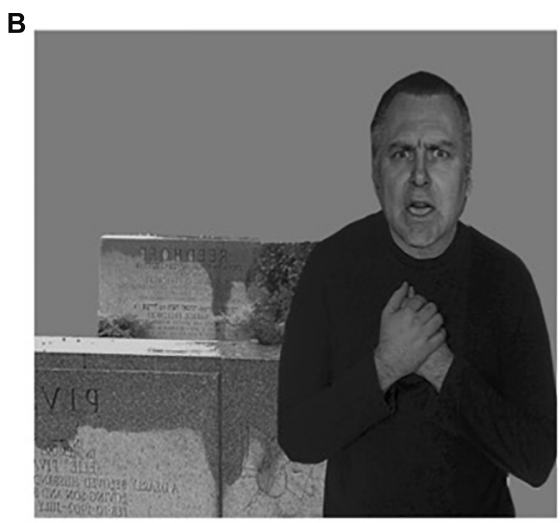

FIGURE 2 | Examples of stimuli of task face-body context. (A) Trial congruent face-anger and body-anger. (B) Trial incongruent face-anger and body-sadness. Permission obtained from Aviezer et al. (2008).

and $100 \mathrm{RPM}$, and active recovery at 75\% HRmax. Phase 3, Final recovery (5 $\mathrm{min}$ ) 60\% HRmax between 50 and 70 RPM (See examples of the planimetry - additional material). The group control stayed at rest for $45 \mathrm{~min}$ in the facilities of the fitness center. HR measures were the following, HRrest $(M=65.4, D E=9.8)$, HRmean $(M=136.8, D E=12.3)$, HRmax $(M=186.1 D E=9.5)$.

Experiment 2 - Sedentary group: The participants were selected randomly to the experimental and control group (see Figure 1). The experimental group performed an indoor cycling session of 35 min duration with intensities of 75 and $85 \%$ HRmax (see Supplementary Figure S1). The protocol had three phases. Phase 1, warming up and muscle activation (7 $\mathrm{min}$ ) 65-75\%HRmax between 70 and 90RPM. Phase 2, main work (17 min) with four sections of 4 min with peaks of $85 \% \mathrm{HRmax}$ between 50 and 100RPM. Phase 3, final recovery (5 min) 75-65\%HRmax between 90 and 70RPM. The control group remained at rest for $30 \mathrm{~min}$ after the post-test. HR measures were, HRrest $(M=63.1, D E=10.1)$, HRmean $(M=143.1, D E=5.7)$, HRmax $(M=185.4, D E=9.5)$. In both experimental groups, the musical track was according to the RPM, intensity, intervals, and commands of the instructor (e.g., position three, sitting, etc.).

\section{Post-experimental Session}

The participants from both experiments had an individual recovery with the monitoring of resting HR. Further, each of them got hydrated during and after PE session. The post-test measure protocols were obtained after getting the resting HR.

\section{Data Analysis}

Both experiments were subjected to the following statistic procedure. The intention-to-treat principle was not carried out, therefore, per-protocol analysis was performed with those who completed the indoor cycling session (see Figure 1). An exploratory analysis of data was executed by calculating tendency central measures (M), of dispersion (SD) and Confidence interval ( $95 \%$ from the mean). Outliers that were observed in the box diagram and located below or above from the interquartile range (Q3-Q1). In these cases, each datum was analyzed by identifying the information gathering process. To some of them, we applied Winsorizing technique, which consists of adjusting cognitive measures and emotional recognition. Three extreme cases were excluded. Each experiment analysis was executed separately in order to estimate and control the effect of every physical condition (active and inactive). Normality Kolmogorov-Smirnov assumptions were confirmed $(n>50)$ for experiment 1 , PA $(p>0.05)$, and Shapiro Wilk $(n<50)$ in experiment 2 , physically inactive $(p>0.05)$. The comparison of demographic, cognitive, physical activity, $\mathrm{HR}$, anxiety, and depression variables were estimated with the $T$ student test for independent samples. The contrast of the acute PE effect was performed by a Mixed Factorial ANOVA or by partially repeated measures. The dependent variables were treated separately due to the levels and conditions of analysis, which each one of these measures has (e.g., execution time, right choices). Finally, four factorial models were estimated.

(i) The first model was $4 \times 2 \times 2$ for the stroop: four Stroop conditions (Stroop-A right choices, Stroop-A time, Stroop$B$ right choices, Stroop-B time) $\times 2$ measures (pre-testpost-test $) \times$ two groups (Experimental and control).

(ii) The second model $2 \times 2 \times 2$ analyzed TMT measures: two TMT conditions (parts A and B), $\times$ two measures (pre-testpost-test $) \times$ two groups (Experimental and Control).

(iii) The third model $2 \times 2 \times 2$ analyzed fluency measures with verbal fluency: (phonological " $S$ " and semantic "animals").

(iv) The fourth model $3 \times 2 \times 2$ analyzed emotional recognition hits: three responses rate (face, body, and errors) $\times$ two measures (pre-test - post-test) $\times$ two groups (Experimental and Control).

The sphericity of each model was corrected through Greenhouse-Geisser. The meaning of interactions and principal effects were analyzed with the post hoc Bonferroni test. The size of the effect was estimated with Eta to the partial square. 


\section{Ethics Statement}

We followed the Helsinki's declaration (WMA, 2013) and the Universal Declaration of Ethic Principles for Psychologist regulations (IUPS, 2008). The evaluation and intervention protocol was approved by the Ethics' Committee of Health Sciences Department of Universidad de Caldas (CBCS-048 Code, Record 015 of 2017) which is coherent with the rules of the Republic of Colombia (Ministerio de Salud República de Colombia, 1993). All the participants signed the informed consent acknowledging the purposes, phases, and possible risks of the research. During the phase of experimental manipulation, there were specialized professionals for medical attention if needed.

\section{RESULTS}

Descriptive data of the PA participants (experiment 1) can be observed in Table 2, and for the physically inactive participants (experiment 2) in Table 3.

\section{Performance Stroop Task}

The Mix Anova $4 \times 2 \times 2$ (Stroop, time, and group conditions) was not significant in the interaction, $[F(1.54,80.5)=452$, $\left.p=0.588, \eta p^{2}=0.009\right]$. The stroop and time conditions (pretest and posttest) were significant $[F(1.54,80.5)=452, p=0.000$, $\left.\eta p^{2}=0.33\right]$. The post-hoc analysis (See Figure 3) showed favorable significant differences for the indoor cycling group in the percentage of right answers of the Stroop-A condition between the pretest and the posttest $(p=0.002)$. In the same way, for the control group, significant changes were observed in the response time of the Stroop-A condition between pretest and posttest $(p=0.009)$, also the answering time of the condition of the Stroop-B, the control group was faster compared to the indoor cycling group $(p=0.001)$.

On the other hand, in the experiment 2 of the PI, the interaction conditions of the stroop, time and group was not significant $\left[F(1.00,57.4)=1.44, p=0.245, \eta p^{2}=0.041\right]$. The time and stroop conditions showed significant differences $\left[F(1.00,57.4)=5.15, p=0.004, \eta p^{2}=0.121\right]$. The post-hoc median comparison, indicated that the indoor cycling group reduced the answering time of stroop-A between the pretest and posttest $(p=0.014)$. On the other side, the control group showed better right answer percentage of stroop-B between the pretest and posttest $(p=0.014)$ (possibly due to an instrumentation effect).

\section{Performance TMT-A/B}

Task performance of TMT A/B, measures (pre-test and posttest) and the group were not significant $[F(1.00,52.0)=0.709$, $\left.p=0.403, \eta p^{2}=0.013\right]$. Mean comparison indicated that the indoor-cycling group obtained a better average performance than the control group. However, these data were not significant (see Figure 4). The post hoc analysis of means Bonferroni comparison showed differences in the pre-test and post-test measures in both TMT-A/B conditions, and in both groups $(p<0.000$, see Figures $4 \mathrm{~A}, \mathrm{~B}$ ).

In experiment 2, significant differences in the interaction between TMT-A/B conditions, measures, and the group were identified $\left[F(1.00,34.0)=0.199, p=0.658, \eta p^{2}=0.006\right]$. The interaction between the TMT-A/B conditions and the measures was significant $\left[F(1.00,34.0)=6.182, p=0.018, \eta p^{2}=0.154\right]$ (see Figures 4E,F). Although the response time averages were better in the indoor-cycling than the control group, these averages were not significant in both TMT-A/B conditions $(p<0.5)$. The post hoc comparison identifies differences of

TABLE 2 | Descriptive dates physically active (PA) - Experiment 1.

\begin{tabular}{|c|c|c|c|c|c|c|c|c|}
\hline \multirow[t]{2}{*}{ Variable } & \multicolumn{4}{|c|}{ Indoor cycling PA $n=25$} & \multicolumn{4}{|c|}{ Control PA $n=29$} \\
\hline & \multicolumn{2}{|c|}{ Pre-test } & \multicolumn{2}{|c|}{ Post-test } & \multicolumn{2}{|c|}{ Pre-test } & \multicolumn{2}{|c|}{ Post-test } \\
\hline \multicolumn{9}{|l|}{ Stroop condition A } \\
\hline Accuracy response (\%) & 95.0 & 4.0 & 97.5 & 2.2 & 97.1 & 3.6 & 97.6 & 2.4 \\
\hline Accuracy response (\%) & 98.7 & 1.9 & 97.1 & 8.7 & 98.6 & 1.7 & 99.0 & 1.5 \\
\hline Response time & 73.4 & 14.3 & 68.7 & 11.8 & 67.0 & 12.3 & 58.8 & 8.8 \\
\hline \multicolumn{9}{|l|}{ Trail making test } \\
\hline TMT-A time (seg) & 51.6 & 15.9 & 36.7 & 16.2 & 55.6 & 21.7 & 44.0 & 21.8 \\
\hline TMT-B time (seg) & 91.1 & 43.2 & 66.1 & 22.3 & 90.7 & 30.6 & 77.1 & 27.3 \\
\hline$\%$ Response face & 42.3 & 12.7 & 44.9 & 14.5 & 44.5 & 11.0 & 46.1 & 12.9 \\
\hline \% Response body & 16.75 & 10.3 & 15.4 & 6.1 & 18.7 & 11.5 & 14.0 & 5.0 \\
\hline$\%$ Error & 40.6 & 12.9 & 36.2 & 11.7 & 39.8 & 10.9 & 39. & 10.7 \\
\hline
\end{tabular}


TABLE 3 | Descriptive dates physically inactive (PI) - Experiment 2.

\begin{tabular}{|c|c|c|c|c|c|c|c|c|}
\hline \multirow[t]{3}{*}{ Variable } & \multicolumn{4}{|c|}{ Indoor cycling PI $n=10$} & \multicolumn{4}{|c|}{ Control group PI $n=26$} \\
\hline & \multicolumn{2}{|c|}{ Pre-test } & \multicolumn{2}{|c|}{ Post-test } & \multicolumn{2}{|c|}{ Pre-test } & \multicolumn{2}{|c|}{ Post-test } \\
\hline & $M$ & $S D$ & $M$ & $S D$ & $M$ & $S D$ & $M$ & $S D$ \\
\hline \multicolumn{9}{|l|}{ Stroop condition A } \\
\hline Accuracy response (\%) & 95.1 & 4.7 & 98.6 & 1.4 & 94.7 & 7.7 & 97.8 & 2.8 \\
\hline Response time (seg) & 81.9 & 19.1 & 68.2 & 17.4 & 70.0 & 14.8 & 66.9 & 12.5 \\
\hline \multicolumn{9}{|l|}{ Stroop condition B } \\
\hline Accuracy response (\%) & 98.2 & 2.5 & 98.3 & 1.6 & 98.7 & 1.9 & 98.9 & 2.4 \\
\hline Response time (seg) & 67.7 & 16.5 & 64.0 & 8.4 & 67.9 & 14.5 & 62.2 & 16.1 \\
\hline \multicolumn{9}{|l|}{ Trail making test } \\
\hline TMT-A time (seg) & 46.3 & 10.9 & 31.7 & 10.7 & 45.7 & 18.8 & 38.5 & 17.4 \\
\hline TMT-B time (seg) & 85.6 & 44.6 & 73.5 & 36.9 & 98.2 & 47.9 & 88.2 & 37.7 \\
\hline \multicolumn{9}{|l|}{ Verbal fluency } \\
\hline Phonemic word "S" & 9.1 & 6.1 & 12.0 & 2.7 & 11.8 & 4.4 & 17.9 & 4.4 \\
\hline Semantic animal & 20.6 & 2.9 & 20.8 & 3.0 & 12.1 & 4.0 & 19.2 & 4.8 \\
\hline \multicolumn{9}{|l|}{ Emotional recognition } \\
\hline$\%$ Response face & 48.4 & 9.7 & 52.9 & 14.3 & 41.2 & 13.8 & 41.7 & 15.0 \\
\hline \% Response body & 20.8 & 12.4 & 16.1 & 8.1 & 22.6 & 9.8 & 20.9 & 12.2 \\
\hline$\%$ Error & 30.9 & 7.4 & 31.0 & 10.2 & 36.3 & 13.4 & 37.3 & 17.2 \\
\hline
\end{tabular}

improvement in the execution of time between the pre-test and post-test measures in TMT-A/B conditions $(p<0.001$, see Figures 4E,F).

\section{Verbal Fluency}

For experiment 1, verbal fluency conditions (greater number of words with the letter " $S$ " and semantic category of animals), measures and the group were not significant $[F(1,52)=0.057$, $\left.p=0.812, \eta p^{2}=0.001\right]$. The post hoc analysis indicated that the control group mentioned a greater number of the semantic animals category than the indoor-cycling group in the post-test $(p<0.042$, see Figure 4D). The group control showed differences in the pre-test and post-test measures in relation to the " $\mathrm{S}$ " letter $(p<0.010$, see Figure 4C).

Interaction between verbal fluency, time (pre-test and posttest) and group was not significant in the physically inactive participants $\left[F(1.00,4.0)=6.182, p=0.018, \eta p^{2}=0.154\right]$. Although the descriptive data show changes in the times, the post hoc analysis did not identify.

\section{Emotional Recognition}

Experiment 1 indicated that three emotional recognition conditions (\% response face, body and error), measures and the group, were not significant $[F(1,52)=0.553, p=0.547$, $\left.\eta p^{2}=0.011\right]$ (Figures 5A,B,F). Measures comparison, with the post hoc Bonferroni showed significant differences in pre-test and post-test measures $(p<0.32)$ in error percentage reduction in the indoor cycling group (see, Figure 5C).

For experiment 2, the interaction effect between the responses of emotional recognition, measures and the group was not significant $[F(1,34)=0.444, p=0.643$, $\eta p^{2}=0.013$ ] (Figures 5A,B,F). The main effect of response percentage of emotional recognition in three conditions was significant $\left[F(1,34)=8.816, p=0.005, \eta p^{2}=0.206\right]$. Mean post hoc comparison pointed out that the indoorcycling group had a significant reduction of the percentage of body emotion recognition in pre-test and post-test measures $(p=0.028$, see Figure 5E). Descriptively, averages indicate that the right choices percentage of facial recognition favored the indoor cycling group (pretest, $M=48.4, D E=9.7$; post-test, $M=52.9, D E=14.3$, see Figure 5D).

\section{DISCUSSION}

Our main objective was to study the effect of acute PE of indoor cycling with a moderate-high intensity range (75$85 \%$ HRmax) on executive function, and the emotional recognition in healthy people who are $\mathrm{PA}$ and inactive. We have outlined two experiments, each one of them for two conditions, PA (Experiment 1) and inactive (Experiment 2). Overall, we highlight two main results. First, we observed some effects of acute $\mathrm{PE}$ on executive functions with a specific PE tendency of indoor-cycling on physically inactive participants. Second, regarding emotional recognition, the group that was exposed to $\mathrm{PE}$ indoor-cycling from PA participants (Experiment 1) reduced errors significantly. Similarly, the PE indoor-cycling group from physically inactive participants had a higher percentage of right choices in facial recognition.

These findings provide evidence of the differential effect of demographic conditions (physically active and inactive), and experimental manipulations considering the type, intensity, acute $\mathrm{PE}$, duration on executive functions and emotional recognition. Below, we mention main results. 


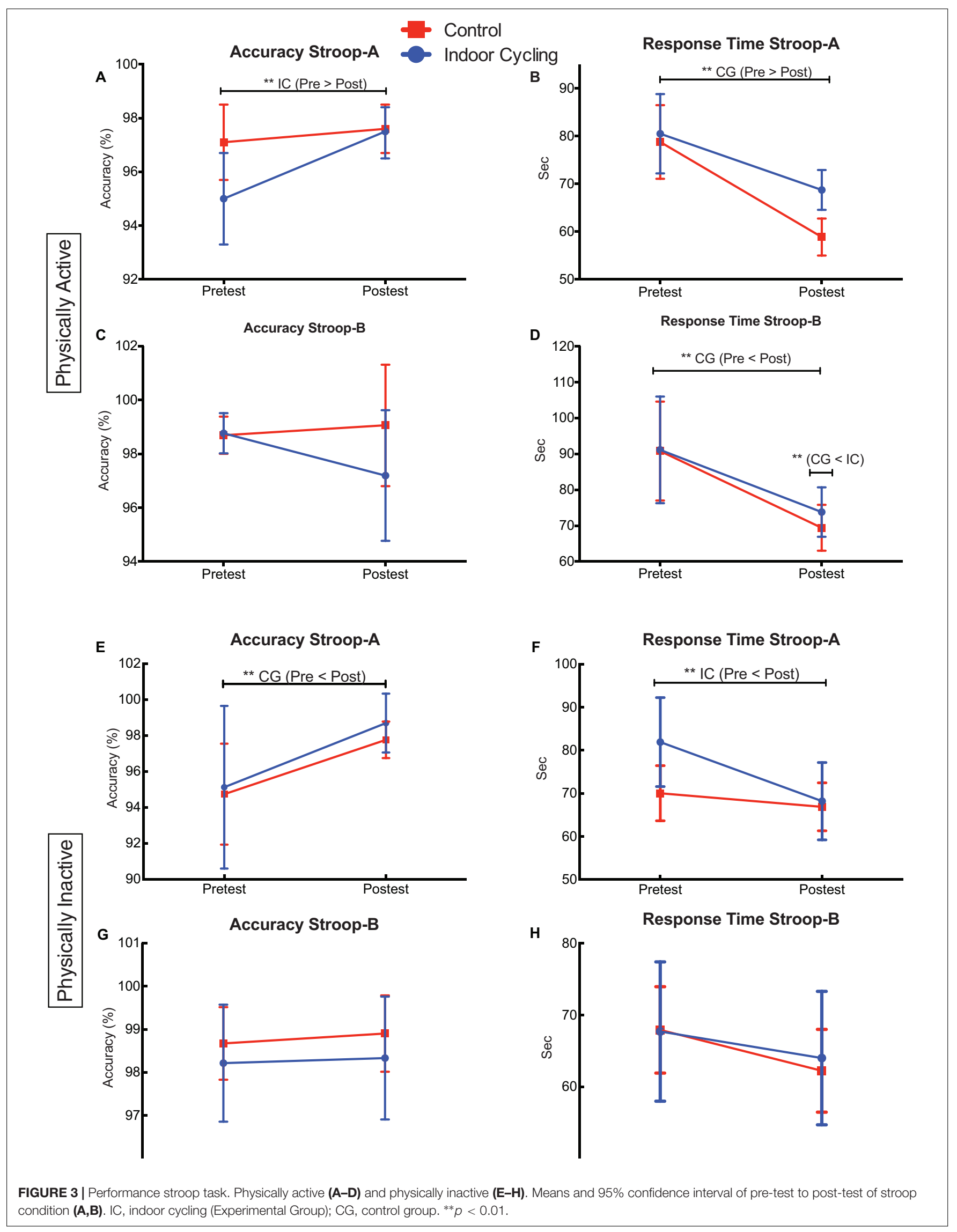




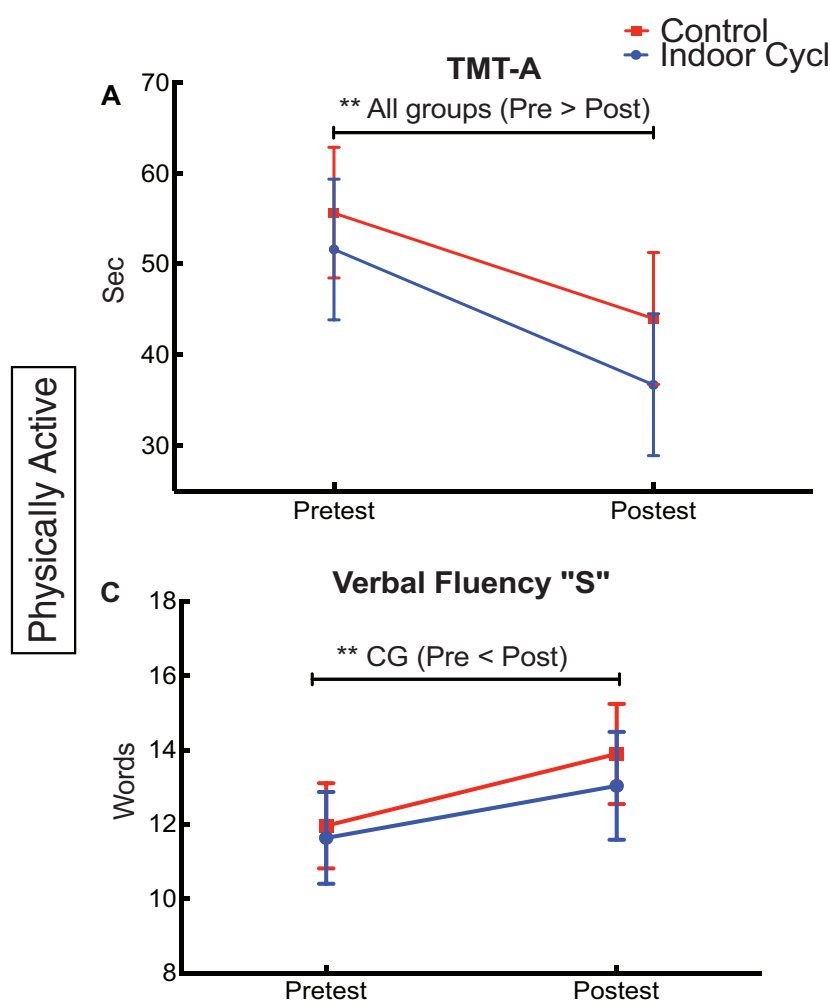

$\begin{array}{rr}\text { B } & 120 \\ 110 \\ 100 \\ 0 \\ \mathbb{d} \\ \text { S } \\ 80 \\ 70 \\ 60\end{array}$
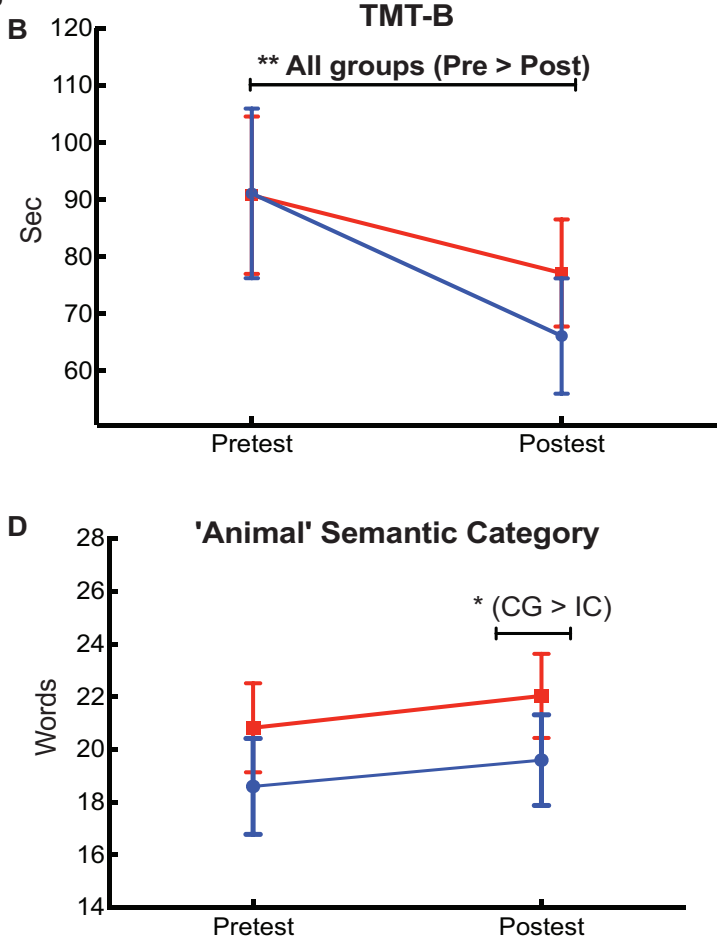

$\mathbf{F}$

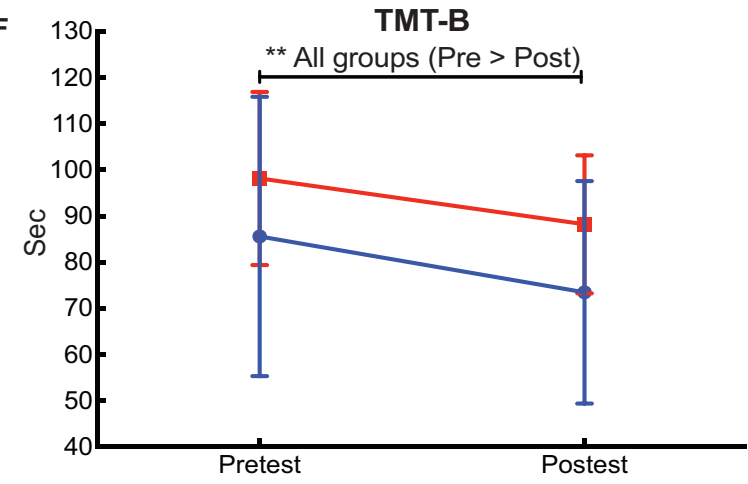

H

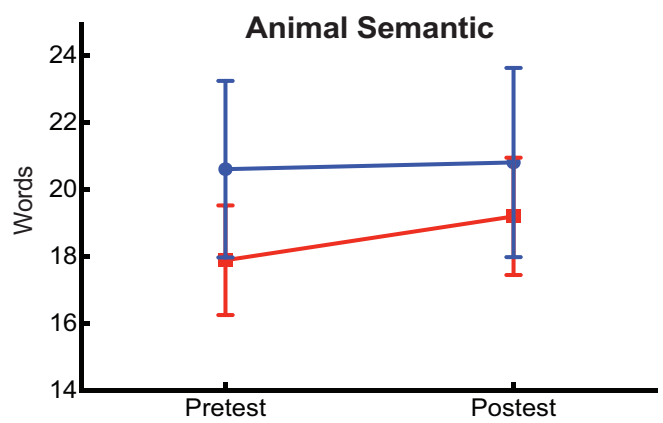

FIGURE 4 | Physically active (A-D) and physically inactive (E-H). Means and 95\% confidence interval of pre-test to post-test of TMT A/B - verbal fluency. IC, Indoor Cycling (Experimental Group); CG, control group. Physically, ${ }^{*} p<0.05$ and ${ }^{* *} p<0.01$. 


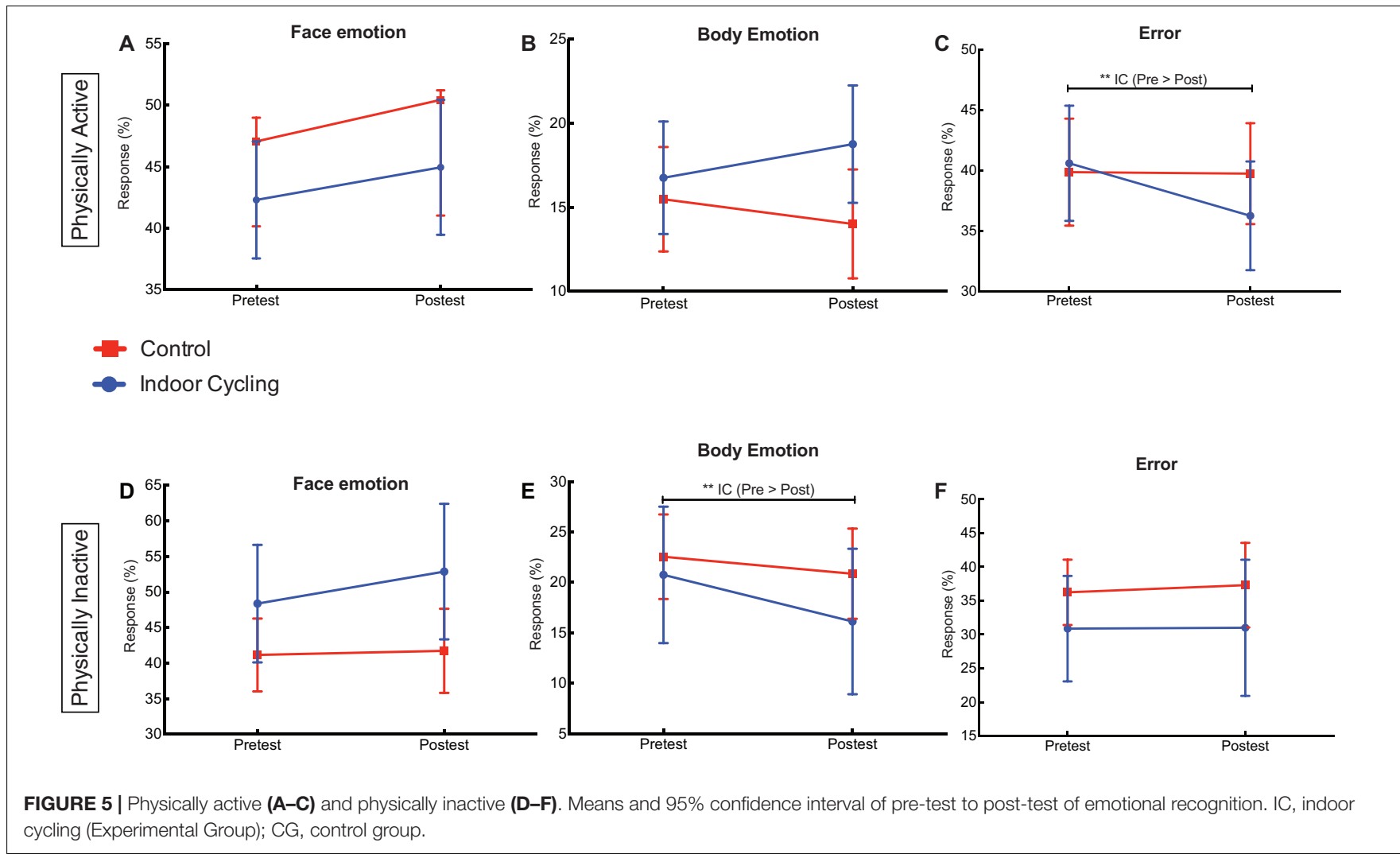

\section{Executive Performance and Physical Exercise (Acute PE)}

The literature that has dealt with the issues of the effect of acute PE on cognitive processes is wide (Tsukamoto et al., 2016; Akram et al., 2018; Quintero et al., 2018; Leyland et al., 2019; Wang et al., 2019b). In both experiments, we worked with a widely used battery on the executive function evaluation at clinic and research level: Stroop, TMT, and Verbal Fluency.

In this way, Experiment 1 data (PA) indicated that the group, which was subjected to $\mathrm{PE}$ indoor cycling, improved significantly in the Stroop-A right choices between pre and post compared to the control group. On the other hand, in the second experiment (physically inactive), the indoor cycling group improved response time in the post-test Stroop-A after the 30-min session (75-85\%HRmax). Regarding the control group, Stroop-A right choices in pre-test and post-test measures and response time improved significantly compared to the indoorcycling group.

In respect of TMT-A/B execution, significant changes enhanced for both groups, which can be explained more by a learning effect of the task than an acute differentiating effect of PE. Regarding phonological and semantic fluency, effects of PE were not observed, but the control group was significantly better. This result must be assumed with reservation on the grounds that this study only presented behavioral measures. Other types of measures and with greater precision can shed light on executive functions and semantic processing. For instance, current contributions point out that the acute PE is associated with greater neuron activation in older adults (Won et al., 2019). The neuron activation and underlying process activation of semantic memory, which is useful for the task performance of semantic processes, which constitutes a promising line of research for future studies (Szepietowska, 2019).

The prior findings are considered from three perspectives. (i) The best performance of the control group, which is PA in the performance of some executive tasks after acute PE, can be explained by a possible physical-cognitive reserve. Thus, optimum cognitive resources would be involved at execution time of the post-test task (Gajewski and Falkenstein, 2015), whereas the indoor cycling group could experiment tiredness due to moderate-high intensities of the HRmax. (ii) From a life cycle perspective, young adults tend to have a relatively stable an executive performance, different from other populations such as children and older adults. Hence, in childhood and old age greater sensibility of executive functions and toward stimuli such as the PE can be evidenced (Ludyga et al., 2016). The prior motive can explain that direct effects are not estimated. (iii) Lastly, the assumption of the hypothesis of the inverted $U$ (Gutin, 1973) indicates that cognitive performance is diminished under high intensities of PE. Some effects of our data could provide evidence to the statement of the inverted U. Another part of our significant outcomes, however, provided evidence that transition of moderate-high intensities has also positive effects on cognitive performance.

Therefore, current studies have reported that high intensities of $\mathrm{PE}$ have a positive effect on executive functions in the 
performance of tasks such as the Stroop and attentional tasks of cancelation (Tsukamoto et al., 2016; Quintero et al., 2018). Additionally, the effect of strong acute PE may last from $30 \mathrm{~min}$ to $2 \mathrm{~h}$ after EP session (Basso et al., 2015). While it is true that the hypothesis of inverted $\mathrm{U}$ has a wide scientific evidence (Pontifex et al., 2009; Chang et al., 2014; De Souto Barreto et al., 2016; Ludyga et al., 2016), the discussion is still open in order to have more solid evidence.

Considering the above-mentioned, studying cognitive processes with great complexity tasks and that are coherent with the postulates of embodied cognition sets up a research line to explore. Specifically, simple tasks of cognitive evaluation such as the ones we implemented, and despite being widely used, have some limitations. The ceiling effect has a high rate of right choices with no variability measures, which is characteristic of a healthy neurologically population (McMorris et al., 2016; Mora-Gonzalez et al., 2019). This is a possible cause that justifies why high intensities of PE and healthy population are not sensitive to the performance of simple cognitive tasks.

Thereupon, qualification of tasks with higher complexity, which can underlie motor and cognitive processes, is an important challenge (e.g., Paradigms of Cognitive Motor Interference by using dual tasks). In such way, if the precision with tasks is evaluated, which involve all the body, similar to sport performance, those conditions can generate an intense effect of PE on cognitive processes (McMorris et al., 2016; Browne et al., 2017). In other words, associating tasks of cognitive complexity that involve the body, such as tasks that have an embodied cognition perspective, is a methodological and scientific challenge to be explored.

\section{Emotional Recognition: Hypothesis of Physical Exercise as a Modulator of Social Cognition}

In accordance with our revision, the studies that deal with the modulating effect of PE on emotional recognition are limited. In this sense, such issue has been addressed in our study. The found data proved that groups exposed to in one indoor-cycling sessions, from both experiments with moderate-high intensities (HRmax), had an effect on the right choices of emotional recognition in context. Thus, this finding suggests that the acute $\mathrm{PE}$, apart from having cognitive benefits, which were previously mentioned, seems to have a direct effect on recognition emotional processing, and mainly, on tasks that require processing of contextual information.

Studies, which are carried out by magnetic functional resonance (fMRI), have demonstrated that body emotional posture influence in facial expression processing (PoyoSolanas et al., 2018). Moreover, it has been suggested that the regions that are in charge of processing dynamic body movements are linked to emotional and body recognition (Kumfor et al., 2018). Using tasks of the same kind of our study, emotional recognition patterns in context in teenagers with criminal conduct have been explored (SantamaríaGarcía et al., 2019), and with patients who suffer from frontotemporal dementia (Kumfor et al., 2018). These processes of body-face recognition are related to brain regions, as the fusiform gyrus (Committeri et al., 2007; Yogev-Seligmann et al., 2008), cingulate cortex, superior temporal gyrus, motor areas, and cerebellum. The identification of body and contextual information is very relevant for emotional recognition (De Gelder, 2006; Aviezer et al., 2012; Poyo-Solanas et al., 2018).

To sum up, our results are evidence that supports the modulator role hypothesis of PE in social cognition processes. Also, they let us put in perspective a path for new research lines, which result promising and hopeful so as to enhance the quality of life of people.

\section{Implications, Limitation, and Future Research Directions}

In agreement with the aforementioned, our results have practical implications, which are directly related to the quality of life of people. They are also pertinent for how the real world functions due to the easy access to $\mathrm{PE}$ in relation to its cost-benefit and non-invasiveness. The inclusion of programs of health promotion through the $\mathrm{PE}$ in school ages, and preventions of diseases in adulthood as a strategy to possible neurodegenerative disease emergence, hence, potential strategies of intervention at a preventive and psychoeducational level (Aguirre-Loaiza et al., 2019). Likewise, instructors and practitioners can train PA and inactive people through intervals of moderate-high intensities as part of a conditioning program as well as parallel purposes that are related with the objective of improving their cognitive processes.

On the other hand, and from a theoretical perspective, our results contribute to the increasing body of knowledge that studies modulator effect comprehension of acute PE on cognitive processes (Pontifex et al., 2019). Additionally, this study considered for the first time the immediate effect of PE in the assessment of emotional-context recognition, its huge scope and potential benefits in social human relationships. In this way, the path for future works in the line of social cognition is marked, modulating the cognitive and brain processes through PE.

This study, however, had some limitations. Firstly, physical activity measures were self-reported. The instruments use, such as the IPAQ-SF in the valoration and allocations to the experiments, was the first limitation. Therefore, more objective evaluations through aerobic conditions will allow a higher control, which is an important criterion for future studies. Secondly, our study kept isolated analysis between two experiments so as to identify a direct effect of duration and PE session intensities. Thus, it would be interesting that both sample conditions are compared (physically active and inactive) in a further analysis. Although it seems that physical condition is not a conditional requirement for temporary improvements and PE benefits (Ludyga et al., 2016). Other works, though, insist on considering physical (Labelle et al., 2013). Finally, our study worked with a single conditional capacity; there 
are other capacities to be studied (e.g., strength, coordination, among others). Indeed, it is possible the combination of these capacities pose an important work line yet to be studied. Likewise, manipulating a chronic PE program and linking cognitive tasks with complex paradigms is a limitation that if addressed in new proposals, it may clarify of the modulating role of PE. These results should be considered with caution due to the sample size, therefore new work is encouraged (Slade et al., 2016).

After mentioning the limitations, we deem that future studies can address: (i) research that demonstrates the role of EP with different intensities. (ii) Apart from the prior, the assessment and design of other measures of cognitive domains, which are associated to the social cognition and embodied cognition with greater complexity, allowing higher sensibility and specificity. For instance, dual tasks in which the body performance is linked. (iii) Effect exploration of other conditional capacities (e.g., coordination). (iv) Motor expertise exploration and analysis of the cognitivephysical reserve relation regarding the cognitive-emotional performance in context.

\section{CONCLUSION}

In summary, our findings add evidence in which the acute PE with moderate-high intensities of HRmax (75-85\%) has positive effects on executive functions, and mainly, in the emotionalcontextual recognition.

\section{DATA AVAILABILITY STATEMENT}

The datasets generated for this study are available on request to the corresponding author.

\section{ETHICS STATEMENT}

The research was approved by the Ethics Committee of the School of Health Sciences, Caldas University (Code CBCS-048, Acta015 from 2017). All the participants signed the informed agreement and knew the purposes of the investigation and

\section{REFERENCES}

ACSM, (2018). Guidelines for Exercise Testing and Prescription, 10th Edn. Philadelphia: Wolters Kluwer Health.

Aguirre-Loaiza, H. H., Parra, J., Bartolo, L., Cardona, M. A., and Arenas, J. A. (2019). Desempeño neuropsicológico e indicadores de frecuencia, duración y tiempo de la sesión del ejercicio físico. Pensam. Psicol. $17,19-32$.

Akram, M., Ghous, M., Tariq, I., Khan, H., Paracha, M., and Hussain, B. (2018). The association between physical activity with cognitive and cardiovascular deconditioning in age related decline of college students. JPMA. J. Pakistan Med. Assoc. 68, 1755-1758.

Armstrong, N., and Welsman, J. R. (2007). Aerobic fitness: what are we measuring? Med. Sport Sci. 50, 5-25. doi: 10.1159/000101073

Aviezer, H., Bentin, S., Dudarev, V., and Hassin, R. R. (2011). The Automaticity of emotional face-context integration humans are tuned to recognize facial its respective phases. The patients/participants provided their written informed consent to participate in this study.

\section{AUTHOR CONTRIBUTIONS}

HA-L, JA, IA, AF-J, SR-B, and FA-Z conceived and designed the experiments. JA, IA, SB-G, and H-AL collected data. HA-L and JA performed the statistical analysis. AF-J, CN, SB-G, HA-L, and $A G-M$ interpreted the data. HA-L, AF-J, and SB-G wrote the original draft. HA-L, JA, IA, AF-J, SB-G, SR-B, FA-Z, CN, and $A G-M$ reviewed, edited and drafted the manuscript, and approved the final version.

\section{FUNDING}

This work was supported by Universidad Católica de Pereira (DII-016-020/2017) and Universidad de Caldas. This work was also supported in part by Universidad de Medellín to $\mathrm{CN}$ and Universidad Cooperativa de Colombia - Pereira to SB-G.

\section{ACKNOWLEDGMENTS}

We thank the participants of study, Psychology Program students, Ing. Daniela Torres of Universidad Católica de Pereira, Physical Education Program students of Universidad del Quindío, Prof. Leonardo Álzate, and Bodytech - Armenia, Colombia.

\section{SUPPLEMENTARY MATERIAL}

The Supplementary Material for this article can be found online at: https://www.frontiersin.org/articles/10.3389/fpsyg. 2019.02774/full\#supplementary-material

FIGURE S1 | Session Indoor Cycling - Planimetry.

TABLE S1 | Consensus on Exercise Reporting Template.

expressions of emotion in a fast. Hassin Trope 11, 1406-1414. doi: 10.1037/ a0023578

Aviezer, H., Hassin, R. R., Ryan, J., Grandy, C., Susskind, J., Anderson, A., et al. (2008). Angry, disgusted, or afraid? Studies on the malleability of emotion. Psychol. Sci. 19, 724-732. doi: 10.1111/j.1467-9280.2008.02148.x

Aviezer, H., Trope, Y., and Todorov, A. (2012). Body cues, not facial expressions, discriminate between intense positive and negative emotions. Science 338, 1225-1229. doi: 10.1126/science. 1224313

Basso, J. C., Shang, A., Elman, M., Karmouta, R., and Suzuki, W. A. (2015). Acute exercise improves prefrontal cortex but not hippocampal function in healthy adults. J. Int. Neuropsychol. Soc. 21, 791-801. doi: 10.1017/S135561771500106X

Basso, J. C., and Suzuki, W. A. (2017). The effects of acute exercise on mood, cognition, neurophysiology, and neurochemical pathways: a review. Brain Plast. 2, 127-152. doi: 10.3233/BPL- 160040

Borkowski, J., Benton, A. L., and Spreen, O. (1967). Word fluency and brain damage. Neuropsychologia 5, 135-140. doi: 10.1016/0028-3932(67)90015-2 
Brand, S., Gerber, M., Colledge, F., Holsboer-Trachsler, E., Pühse, U., and Ludyga, S. (2019). Acute exercise and emotion recognition in young adolescents. J. Sport Exerc. Psychol. 41, 129-136. doi: 10.1123/jsep.2018-0160

Browne, S. E., Flynn, M. J., O’Neill, B. V., Howatson, G., Bell, P. G., and HaskellRamsay, C. F. (2017). Effects of acute high-intensity exercise on cognitive performance in trained individuals: a systematic review. Progr. Brain Res. 234, 161-187. doi: 10.1016/bs.pbr.2017.06.003

Chang, Y.-K., Chi, L., Etnier, J. L., Wang, C.-C., Chu, C.-H., and Zhou, C. (2014). Effect of acute aerobic exercise on cognitive performance: role of cardiovascular fitness. Psychol. Sport Exerc. 15, 464-470. doi: 10.1016/J.PSYCHSPORT.2014. 04.007

Chang, Y.-K., Chu, C.-H., Wang, C.-C., Song, T.-F., and Wei, G.-X. (2015). Effect of acute exercise and cardiovascular fitness on cognitive function: an eventrelated cortical desynchronization study. Psychophysiology 52, 342-351. doi: 10.1111/psyp. 12364

Chang, Y.-K., Labban, J. D., Gapin, J. I., and Etnier, J. L. (2012). The effects of acute exercise on cognitive performance: a meta-analysis. Brain Res. 1453, 87-101. doi: 10.1016/j.brainres.2012.02.068

Chen, P. Y., and Chang, H.-C. (2012). The coping process of patients with cancer. Eur. J. Oncol. Nurs. 16, 10-16. doi: 10.1016/j.ejon.2011.01.002

Committeri, G., Pitzalis, S., Galati, G., Patria, F., Pelle, G., Sabatini, U., et al. (2007). Neural bases of personal and extrapersonal neglect in humans. Brain 130, 431-441. doi: 10.1093/brain/awl265

Craig, C. L., Marshall, A. L., Sjöström, M., Bauman, A. E., Booth, M. L., Ainsworth, B. E., et al. (2003). International physical activity questionnaire: 12-country reliability and validity. Med. Sc. Sports Exerci. 35, 1381-1395. doi: 10.1249/01. MSS.0000078924.61453.FB

De Gelder, B. (2006). Towards the neurobiology of emotional body language. Nat. Rev. Neurosci. 7, 242-249. doi: 10.1080/10915810305084

De Souto Barreto, P., Delrieu, J., Andrieu, S., Vellas, B., and Rolland, Y. (2016). Physical activity and cognitive function in middle-aged and older adults. Mayo Clin. Proc. 91, 1515-1524. doi: 10.1016/j.mayocp.2016.06.032

Donnelly, J. E., Hillman, C., Castelli, D. M., Etnier, J. L., Lee, S., Tomporowski, P., et al. (2016). Physical activity, fitness, cognitive function, and academic achievement in children: a systematic review. Med. Sci. Sports Exerc. 48, 11971222. doi: 10.1249/MSS.0000000000000901.Physical

Erickson, K. I., Hillman, C., and Kramer, A. F. (2015). Physical activity, brain, and cognition. Curr. Opin. Behav. Sci. 4, 27-32. doi: 10.1016/j.cobeha.2015.01.005

Esteban-Cornejo, I., Rodriguez-Ayllon, M., Verdejo-Roman, J., Cadenas-Sanchez, C., Mora-Gonzalez, J., Chaddock-Heyman, L., et al. (2019). Physical fitness, white matter volume and academic performance in children: findings from the ActiveBrains and FITKids2 projects. Front. Psychol. 10:208. doi: 10.3389/fpsyg. 2019.00208

Etnier, J. L., Drollette, E. S., and Slutsky, A. B. (2019). Physical activity and cognition: a narrative review of the evidence for older adults. Psychol. Sport Exerci. 42, 156-166. doi: 10.1016/J.PSYCHSPORT.2018.12.006

Florez, J. C., Ostrosky, F., and Lozano, A. (2012). BANFE - Batería Neuorpsicológica de Funciones Ejecutivas y Lóbulos Frontales. México DF: Manual Moderno.

Forsén, L., Loland, N. W., Vuillemin, A., Chinapaw, M. J. M., van Poppel, M. N. M., Mokkink, L. B., et al. (2010). Self-administered physical activity questionnaires for the elderly. Sports Med. 40, 601-623. doi: 10.2165/11531350-00000000000000

Gajewski, P. D., and Falkenstein, M. (2015). Long-term habitual physical activity is associated with lower distractibility in a Stroop interference task in aging: behavioral and ERP evidence. Brain Cogn. 98, 87-101. doi: 10.1016/j.bandc. 2015.06.004

Gil, L., Ruiz De Sánchez, C., Gil, F., Romero, S. J., and Pretelt Burgos, F. (2015). Validation of the montreal cognitive assessment (MoCA) in Spanish as a screening tool for mild cognitive impairment and mild dementia in patients over 65 years old in Bogotá, Colombia. Int. J. Geriat. Psychiatry 30, 655-662. doi: 10.1002/gps.4199

Gunnell, K. E., Poitras, V. J., LeBlanc, A., Schibli, K., Barbeau, K., Hedayati, N., et al. (2018). Physical activity and brain structure, brain function, and cognition in children and youth: a systematic review of randomized controlled trials. Ment. Health Phys. Act. 16, 105-127. doi: 10.1016/J.MHPA.2018. 11.002

Guthold, R., Stevens, G. A., Riley, L. M., and Bull, F. C. (2018). Worldwide trends in insufficient physical activity from 2001 to 2016: a pooled analysis of 358 population-based surveys with 1.9 million participants. Lancet. Global Health 6, e1077-e1086. doi: 10.1016/S2214-109X(18)30357-7

Gutin, B. (1973). Exercise-induced activation and human performance: a review. Res. Q. Am. Assoc. Health Phys. Educ. Recreat. 44, 256-268. doi: 10.1080/ 10671188.1973.10615204

Hagger, M. S. (2019). Habit and physical activity: theoretical advances, practical implications, and agenda for future research. Psychol. Sport Exerc. 42, 118-129. doi: 10.1016/J.PSYCHSPORT.2018.12.007

Herting, M. M., Colby, J. B., Sowell, E. R., and Nagel, B. J. (2014). White matter connectivity and aerobic fitness in male adolescents. Dev. Cogn. Neurosci. 7, 65-75. doi: 10.1016/j.dcn.2013.11.003

Herting, M. M., and Nagel, B. J. (2013). Differences in brain activity during a verbal associative memory encoding task in high- and low-fit adolescents. J. Cogn. Neurosci. 25, 595-612. doi: 10.1162/jocn_a_00344

Hillman, C., Logan, N. E., and Shigeta, T. T. (2019). A review of acute physical activity effects on brain and cognition in children. Transl. J. Am. Sport Med. 4, $132-136$.

Hillman, C., Pontifex, M. B., Castelli, D. M., Khan, N. A., Raine, L. B., Scudder, M. R., et al. (2014). Effects of the FITKids Randomized Controlled Trial on Executive Control and Brain Function. Pediatrics 134, e1063-e1071. doi: 10. 1542/peds.2013-3219

IUPS, (2008). "Universal declaration of ethical principles for psychologists," in Assembly of the International Union of Psychological Science, (Palais de Chaillot: Universal Declaration).

Joyce, J., Smyth, P. J., Donnelly, A. E., and Davranche, K. (2014). The simon task and aging: does acute moderate exercise influence cognitive control? Med. Sci. Sports Exerc. 46, 630-639. doi: 10.1249/MSS.0b013e3182a77980

Karvonen, M., Kentala, E., and Mustala, O. (1957). The effects of training on heart rate; a longitudinal study. Ann. Med. Exp. Biol. Fenn. 35, 307-315.

Kumfor, F., Ibañez, A., Hutchings, R., Hazelton, J. L., Hodges, J. R., and Piguet, O. (2018). Beyond the face: how context modulates emotion processing in frontotemporal dementia subtypes. Brain 141, 1172-1185. doi: 10.1093/brain/ awy002

Labelle, V., Bosquet, L., Mekary, S., and Bherer, L. (2013). Decline in executive control during acute bouts of exercise as a function of exercise intensity and fitness level. Brain Cogn. 81, 10-17. doi: 10.1016/j.bandc.2012.10.001

Lambourne, K., and Tomporowski, P. (2010). The effect of exercise-induced arousal on cognitive task performance: a meta-regression analysis. Brain Res. 1341, 12-24. doi: 10.1016/j.brainres.2010.03.091

Leyland, L.-A., Spencer, B., Beale, N., Jones, T., and van Reekum, C. M. (2019). The effect of cycling on cognitive function and well-being in older adults. Plos One 14:e0211779. doi: 10.1371/journal.pone.0211779

Li, L., Men, W. W., Chang, Y. K., Fan, M. X., Ji, L., and Wei, G. X. (2014). Acute aerobic exercise increases cortical activity during working memory: a functional MRI study in female college students. PLoS One 9:e99222. doi: 10.1371/journal. pone.0099222

Ludyga, S., Gerber, M., Brand, S., Holsboer-Trachsler, E., and Pühse, U. (2016). Acute effects of moderate aerobic exercise on specific aspects of executive function in different age and fitness groups: a meta-analysis. Psychophysiology 53, 1611-1626. doi: 10.1111/psyp.12736

McMorris, T., Turner, A., Hale, B. J., and Sproule, J. (2016). "Beyond the catecholamines hypothesis for an acute exercise-cognition interaction: a neurochemical perspective," in Exercise-Cognition Interaction, ed. T. McMorris, (Amsterdam: Elsevier), 65-103. doi: 10.1016/b978-0-12-800778-5.00004-9

McSween, M. P., Coombes, J. S., MacKay, C. P., Rodriguez, A. D., Erickson, K. I., Copland, D. A., et al. (2019). The immediate effects of acute aerobic exercise on cognition in healthy older adults: a systematic review. Sports Med. 49, 67-82. doi: 10.1007/s40279-018-01039-9

Ministerio de Salud - República de Colombia, (1993). Resolución 8430, 1993. Bogotá: Ministerio De Salud Resolucion $\$:$.

Mora-Gonzalez, J., Esteban-Cornejo, I., Cadenas-Sanchez, C., Migueles, J. H., Molina-Garcia, P., Rodriguez-Ayllon, M., et al. (2019). Physical fitness, physical activity, and the executive function in children with overweight and obesity. J. Pediatr. 208, 50-56. doi: 10.1016/j.jpeds.2018.12.028

Nasreddine, L., Philips, A., Bedirian, V., Charbonneau, S., Whitehead, V., Collin, I., et al. (2005). The montreal cognitive assessment,MOCA:a brief screening tool for mild cognitive impairment. Am. Geriat. Soc. 53, 695-699. doi: 10.1029/ WR017i002p00410 
O'Driscoll, C., and Shaikh, M. (2017). Cross-cultural applicability of the montreal cognitive assessment (MoCA): a systematic review. J. Alzheimer's Dis. 58, 789-801. doi: 10.3233/JAD-161042

Päivärinne, V., Kautiainen, H., Heinonen, A., and Kiviranta, I. (2018). Relations between subdomains of physical activity, sedentary lifestyle, and quality of life in young adult men. Scand. J. Med. Sci. Sports 28, 1389-1396. doi: 10.1111/sms. 13003

Pedraza, O. L., Salaza, A. M., Sierra, F., Soler, D., Castro, J., Castillo, P., et al. (2016). Confiabilidad, validez de criterio y discriminante del montreal cognitive assessment (MoCA) test, en un grupo de adultos de Bogotá. Acta Méd. Colomb. 41, 221-228.

Pedraza, O. L., Sánchez, E., Plata, S. J., Montalvo, C., Galvis, P., Chiquillo, A., et al. (2014). Puntuaciones del MoCA y el MMSE en pacientes con deterioro cognitivo leve y demencia en una clínica de memoria en Bogotá. Acta Neurol. Colomb. 30, 22-31.

Peruyero, F., Zapata, J., Pastor, D., and Cervelló, E. (2017). The acute effects of exercise intensity on inhibitory cognitive control in adolescents. Front. Psychol. 8:921. doi: 10.3389/fpsyg.2017.00921

Pontifex, M. B., Hillman, C., Fernhall, B., Thompson, K. M., and Valentini, T. A. (2009). The effect of acute aerobic and resistance exercise on working memory. Med. Sci. Sports Exerc. 41, 927-934. doi: 10.1249/MSS.0b013e3181907d69

Pontifex, M. B., McGowan, A. L., Chandler, M. C., Gwizdala, K. L., Parks, A. C., Fenn, K., et al. (2019). A primer on investigating the after effects of acute bouts of physical activity on cognition. Psychol. Sport Exerc. 40, 1-22. doi: 10.1016/j.psychsport.2018.08.015

Poyo-Solanas, M., Zhan, M., Vaessen, M., Hortensius, R., Engelen, T., and de Gelder, B. (2018). Looking at the face and seeing the whole body. Neural basis of combined face and body expressions. Soc. Cog. Affect. Neurosci. 13, 135-144. doi: 10.1093/scan/nsx130

Prakash, R. S., Voss, M. W., Erickson, K. I., and Kramer, A. F. (2015). Physical activity and cognitive vitality. Annu. Rev. Psychol. 66, 769-797. doi: 10.1146/ annurev-psych-010814-015249

Quintero, A. P., Bonilla-Vargas, K. J., Correa-Bautista, J. E., Domínguez-Sanchéz, M. A., Triana-Reina, H. R., Velasco-Orjuela, G. P., et al. (2018). Acute effect of three different exercise training modalities on executive function in overweight inactive men: a secondary analysis of the BrainFit study. Physiol. Behav. 197, 22-28. doi: 10.1016/j.physbeh.2018.09.010

Rabin, L., Barr, W., and Burton, L. (2005). Assessment practices of clinical neuropsychologists in the United States and Canada: a survey of INS, NAN, and APA division 40 members. Arch. Clin. Neuropsychol. 20, 33-65. doi: 10.1016/j. acn.2004.02.005

Reed, J., and Ones, D. S. (2006). The effect of acute aerobic exercise on positive activated affect: a meta-analysis. Psychol. Sport Exerc. 7, 477-514. doi: 10.1016/ J.PSYCHSPORT.2005.11.003

Reitan, R. M. (1958). Validity of the trail making test as an indicator of organic brain damage. Percept. Mot. Skills 8, 271-276. doi: 10.2466/pms.1958.8.3.271

Reiter, K., Nielson, K. A., Smith, T. J., Weiss, L. R., Alfini, A. J., and Smith, J. C. (2015). Improved cardiorespiratory fitness is associated with increased cortical thickness in mild cognitive impairment. J. Int. Neuropsychol. Soc. 21, 757-767. doi: 10.1017/S135561771500079X

Sáez de Asteasu, M. L., Martínez-Velilla, N., Zambom-Ferraresi, F., CasasHerrero, Á, and Izquierdo, M. (2017). Role of physical exercise on cognitive function in healthy older adults: a systematic review of randomized clinical trials. Ageing Res. Rev. 37, 117-134. doi: 10.1016/J.ARR.2017. 05.007

Sanda, B., Vistad, I., Haakstad, L. A. H., Berntsen, S., Sagedal, L. R., Lohne-Seiler, H., et al. (2017). Reliability and concurrent validity of the international physical activity questionnaire short form among pregnant women. BMC Sports Sci. Med. Rehabil. 9:7. doi: 10.1186/s13102-017-0070-4

Santamaría-García, H., Ibáñez, A., Montaño, S., García, A. M., PatiñoSaenz, M., Idarraga, C., et al. (2019). Out of context, beyond the face: neuroanatomical pathways of emotional face-body language integration in adolescent offenders. Front. Behav.; Neurosci. 13:1-14. doi: 10.3389/fnbeh.2019. 00034

Sanz, J., and García-Vera, M. P. (2013). Rendimiento diagnóstico y estructura factorial del Inventario para la Depresión de Beck-Segunda Edición (BDI-II) en pacientes españoles con trastornos psicológicos. Anales Psicol. 29, 66-75. doi: 10.6018/analesps.29.1.130532
Silva, B. G. C. D., da Silva, I. C. M., Ekelund, U., Brage, S., Ong, K. K., De Lucia Rolfe, E., et al. (2019). Associations of physical activity and sedentary time with body composition in Brazilian young adults. Sci. Rep. 9:5444. doi: 10.1038/s41598-019-41935-2

Sink, K. M., Espeland, M. A., Castro, C. M., Church, T., Cohen, R., Dodson, J. A., et al. (2015). Effect of a 24-month physical activity intervention vs health education on cognitive outcomes in sedentary older adults. JAMA 314, 781-790. doi: 10.1001/jama.2015.9617

Slade, S. C., Dionne, C. E., Underwood, M., and Buchbinder, R. (2016). Consensus on exercise reporting template (CERT): explanation and elaboration statement. Br. J. Sports Med. 50, 1428-1437. doi: 10.1136/bjsports-2016096651

Strauss, E., Sherman, E. M., and Spreen, O. (2007). A Compendium of Neuropsychological Tests: Administration, Norms, and Commentary. New York, NY: Oxford University Press.

Szepietowska, E. M. (2019). Cognitive reserve as a factor determining the level of cognitive functions in adults: a preliminary report. Psych. Psychol. Kliniczna 19, 32-41. doi: 10.15557/PiPK.2019.0005

Tsukamoto, H., Suga, T., Takenaka, S., Tanaka, D., Takeuchi, T., Hamaoka, T., et al. (2016). Greater impact of acute high-intensity interval exercise on post-exercise executive function compared to moderate-intensity continuous exercise. Physiol. Behav. 155, 224-230. doi: 10.1016/j.physbeh.2015.12.021

Van Poppel, M. N. M., Chinapaw, M. J. M., Mokkink, L. B., van Mechelen, W., and Terwee, C. B. (2010). Physical Activity Questionnaires for Adults. Sports Med. 40, 565-600. doi: 10.2165/11531930-000000000-00000

Vazou, S., Pesce, C., Lakes, K., and Smiley-Oyen, A. (2019). More than one road leads to Rome: a narrative review and meta-analysis of physical activity intervention effects on cognition in youth. Int. J. Sport Exerc. Psychol. 17, 153-178. doi: 10.1080/1612197X.2016.1223423

Vieira, R. A., Costa, E. C., Sales, M. M., Fonteles, A. I., Moraes, J. F. V. N., and Barros, J. D. F. (2016). Efeito agudo do exercício aeróbio vigoroso sobre o controle inibitório em adolescentes. Rev. Paulista Pediat. 34, 154-161. doi: 10.1016/J.RPPED.2015.08.004

Voss, M. W., Soto, C., Yoo, S., Sodoma, M., Vivar, C., and Van Praag, H. (2019). Exercise and hippocampal memory systems. Trends Cogn. Sci. 23, 318-333. doi: 10.1016/j.tics.2019.01.006

Voss, M. W., Weng, T. B., Burzynska, A. Z., Wong, C. N., Cooke, G. E., Clark, R., et al. (2015). Fitness, but not physical activity, is related to functional integrity of brain networks associated with aging. NeuroImage 131, 113-125. doi: 10.1016/j.neuroimage.2015.10.044

Wang, C. H., Moreau, D., Yang, C.-T., Lin, J.-T., Tsai, Y.-Y., and Tsai, C.-L. (2019a). The influence of aerobic fitness on top-down and bottom-up mechanisms of interference control. Neuropsychology 33, 245-255. doi: 10.1037/neu0000507

Wang, C. H., Moreau, D., Yang, C. T., Tsai, Y. Y., Lin, J. T., Liang, W. K., et al. (2019b). Aerobic exercise modulates transfer and brain signal complexity following cognitive training. Biol. Psychol. 144, 85-98. doi: 10.1016/j.biopsycho. 2019.03.012

Wang, Y. P., and Gorenstein, C. (2013). Psychometric properties of the beck depression Inventory-II: a comprehensive review. Rev. Bras. Psiquiat. 35, 416431. doi: 10.1590/1516-4446-2012-1048

Warburton, D., Jamnik, V., Bredin, S., and Gledhill, N. (2011). The physical activity readiness questionnaire for everyone (PAR-Q+) and electronic physical activity readiness medical examination (ePARmed-X+). Health Fit. J. Can. 4, 3-17.

WMA, (2013). Declaracioin de Helsinki de la AMM - Principios Eiticos Para las Investigaciones Meidicas en Seres Humanos. Ferney-Voltaire: World Medical Association, Inc.

Won, J., Alfini, A. J., Weiss, L. R., Michelson, C. S., Callow, D. D., Ranadive, S. M., et al. (2019). Semantic memory activation after acute exercise in healthy older adults. J. Int. Neuropsychol. Soc. 25, 557-568. doi: 10.1017/S135561771900 0171

World Health Organization [WHO], (2007). A Guide for Population-Based Approaches to Increasing Levels of Physical Activity: Implementation of the WHO Global Strategy On diet, Physical Activity and Health. Geneva: WHO.

World Health Organization [WHO], (2010). Global recommendations on physical activity for helth. Geneva: WHO Library Cataloguing-in-Publication.

World Health Organization [WHO], (2018). Why is physical activity important? Available at: https://www.paho.org/hq/index.php?option=com_content\&view 
$=$ article\&id=14459:physical-activity\&Itemid=1969\&lang=en $\quad$ (accessed $\quad$ on February 13, 2019).

Wu, C.-H., Karageorghis, C. I., Wang, C.-C., Chu, C.-H., Kao, S.-C., Hung, T.M., et al. (2019). Effects of acute aerobic and resistance exercise on executive function: an ERP study. J. Sci. Med. Sport 22, 1367-1372. doi: 10.1016/j.jsams. 2019.07.009

Yogev-Seligmann, G., Hausdorff, J. M., and Giladi, N. (2008). The role of executive function and attention in gait. Mov. Disord. 23, 329-342. doi: 10.1002/mds. 21720

Zuniga, K. E., Mueller, M., Santana, A. R., and Kelemen, W. L. (2019). Acute aerobic exercise improves memory across intensity and fitness levels. Memory 27, 628-636. doi: 10.1080/09658211.2018.1546875
Conflict of Interest: The authors declare that the research was conducted in the absence of any commercial or financial relationships that could be construed as a potential conflict of interest.

Copyright (C) 2019 Aguirre-Loaiza, Arenas, Arias, Franco-Jímenez, BarbosaGranados, Ramos-Bermúdez, Ayala-Zuluaga, Núnez and García-Mas. This is an open-access article distributed under the terms of the Creative Commons Attribution License (CC BY). The use, distribution or reproduction in other forums is permitted, provided the original author(s) and the copyright owner(s) are credited and that the original publication in this journal is cited, in accordance with accepted academic practice. No use, distribution or reproduction is permitted which does not comply with these terms. 